PROCEEDINGS OF THE

AMERICAN MATHEMATICAL SOCIETY

Volume 140, Number 2, February 2012, Pages 621-626

S 0002-9939(2011)10928-6

Article electronically published on June 14, 2011

\title{
PICARD NUMBER, HOLOMORPHIC SECTIONAL CURVATURE, AND AMPLENESS
}

\author{
PIT-MANN WONG, DAMIN WU, AND SHING-TUNG YAU
}

(Communicated by Jianguo Cao)

\begin{abstract}
We prove that for a projective manifold with Picard number equal to one, if the manifold admits a Kähler metric whose holomorphic sectional curvature is quasi-negative, then the canonical bundle of the manifold is ample.
\end{abstract}

\section{INTRODUCTION}

Let $M$ be a compact Kähler manifold. Then, it is well known that $M$ is hyperbolic if and only if any holomorphic map $f: \mathbb{C} \rightarrow M$ is a constant. A conjecture of Kobayashi states that if a compact Kähler manifold $M$ is hyperbolic, then its canonical bundle $K_{M}$ is ample (see, for example, [3, p. 370], [2], and 4]). This conjecture clearly holds when $M$ is a compact Riemann surface. For $M$ being a Kähler surface, the conjecture follows from the Enriques-Kodaira classification [2]. Based on the results of Wilson [6], Peternell [4] proved that a 3-dimensional projective hyperbolic manifold has ample canonical bundle, possibly except for certain Calabi-Yau threefolds whose Picard number is not greater than 19.

On the other hand, if a compact Kähler manifold $M$ has strictly negative holomorphic sectional curvature everywhere, then $M$ is hyperbolic. Thus in this paper, we would like to study, under what condition would the negativity of the holomorphic sectional curvature imply the ampleness. As a first step, we consider the manifolds with Picard number equal to 1.

For a Kähler manifold $M$, we say that the holomorphic sectional curvature of $M$ is quasi-negative if the holomorphic sectional curvature is nonpositive everywhere and is strictly negative at one point of $M$. We denote by $\rho(M)$ the Picard number of $M$. Our result is as follows:

Theorem 1. Let $M$ be an $n$-dimensional projective manifold with $\rho(M)=1$. If $M$ admits a Kähler metric $\omega$ whose holomorphic sectional curvature is quasi-negative, then $K_{M}$ is ample.

We remark that the curvature condition in Theorem 1 is sharp; namely, the quasinegativity cannot be replaced by nonpositivity. Indeed, there are 2-dimensional abelian varieties with Picard number equal to 1 ([1, pp. 58-59]).

Our technique is essentially the third author's Schwarz lemma 8] (see also 7]). We incorporate here a trick of Royden [5], which converts the bound of holomorphic sectional curvature to the bound of holomorphic bisectional curvature.

Received by the editors October 12, 2010 and, in revised form, November 30, 2010.

2010 Mathematics Subject Classification. Primary 32Q15, 32Q45, 53C55; Secondary 53C56. 
This paper is based on the discussions of all the authors in the spring of 2010 . The first author, Professor Wong, untimely passed away on July 03, 2010. The second and third authors therefore took up the task of writing the manuscript. The second author would like to thank the warm hospitality of the University of Notre Dame and the support of The Ohio State University.

\section{Proof of THE THEOREM}

Let us first prove the following lemma.

Lemma 2.1 (Royden). Let $(V,\|\cdot\|)$ be a normed vector space over $\mathbb{C}$, and let $R(\mu, \bar{\nu}, \eta, \bar{\xi})$ be a symmetric bi-Hermitian form on $V$; i.e., for all $\mu, \nu, \eta, \xi$ in $V$,

$$
R(\mu, \bar{\nu}, \eta, \bar{\xi})=R(\eta, \bar{\nu}, \mu, \bar{\xi}), \quad R(\nu, \bar{\mu}, \xi, \bar{\eta})=\bar{R}(\mu, \bar{\nu}, \eta, \bar{\xi})
$$

Assume, in addition, that $R$ satisfies

$$
R(\eta, \bar{\eta}, \eta, \bar{\eta}) \leq b\|\eta\|^{4}, \quad \text { for all } \eta \in V,
$$

where $b$ is a constant. Then, for any $m$ orthogonal vectors $\alpha_{1}, \ldots, \alpha_{m}$,

$$
\sum_{i, j=1}^{m} R\left(\alpha_{i}, \bar{\alpha}_{i}, \alpha_{j}, \bar{\alpha}_{j}\right) \leq \frac{b}{2}\left[\left(\sum_{i=1}^{m}\left\|\alpha_{i}\right\|^{2}\right)^{2}+\sum_{i=1}^{m}\left\|\alpha_{i}\right\|^{4}\right] .
$$

Proof. We denote $I=\{1,-1, \sqrt{-1},-\sqrt{-1}\}$. Let

$$
\eta_{\epsilon}=\epsilon_{1} \alpha_{1}+\cdots+\epsilon_{m} \alpha_{m}
$$

where $\epsilon=\left(\epsilon_{1}, \ldots, \epsilon_{m}\right) \in I^{m}$. Clearly, for each $\epsilon \in I^{m}$,

$$
R\left(\eta_{\epsilon}, \bar{\eta}_{\epsilon}, \eta_{\epsilon}, \bar{\eta}_{\epsilon}\right) \leq b\left(\sum_{i=1}^{m}\left\|\alpha_{i}\right\|^{2}\right)^{2}
$$

Thus,

$$
\frac{1}{4^{m}} \sum_{\epsilon \in I^{m}} R\left(\eta_{\epsilon}, \bar{\eta}_{\epsilon}, \eta_{\epsilon}, \bar{\eta}_{\epsilon}\right) \leq b\left(\sum_{i=1}^{m}\left\|\alpha_{i}\right\|^{2}\right)^{2} .
$$

On the other hand, we have, by the symmetry of $R$ and $\sum_{\delta \in I} \delta=0$ and $\sum_{\delta \in I} \delta^{2}=$ 0 , that

$$
\begin{aligned}
& \frac{1}{4^{m}} \sum_{\epsilon \in I^{m}} R\left(\eta_{\epsilon}, \bar{\eta}_{\epsilon}, \eta_{\epsilon}, \bar{\eta}_{\epsilon}\right) \\
& =\frac{1}{4^{m}} \sum_{\epsilon \in I^{m}} \sum_{i, j, k, l=1}^{m} \epsilon_{i} \bar{\epsilon}_{j} \epsilon_{k} \bar{\epsilon}_{l} R\left(\alpha_{i}, \bar{\alpha}_{j}, \alpha_{k}, \bar{\alpha}_{l}\right) \\
& =\sum_{i=1}^{m} R\left(\alpha_{i}, \bar{\alpha}_{i}, \alpha_{i}, \bar{\alpha}_{i}\right)+\sum_{i \neq j}\left[R\left(\alpha_{i}, \bar{\alpha}_{i}, \alpha_{j}, \bar{\alpha}_{j}\right)+R\left(\alpha_{i}, \bar{\alpha}_{j}, \alpha_{j}, \bar{\alpha}_{i}\right)\right] \\
& =\sum_{i=1}^{m} R\left(\alpha_{i}, \bar{\alpha}_{i}, \alpha_{i}, \bar{\alpha}_{i}\right)+2 \sum_{i \neq j} R\left(\alpha_{i}, \bar{\alpha}_{i}, \alpha_{j}, \bar{\alpha}_{j}\right) .
\end{aligned}
$$

It follows that

$$
2 \sum_{i, j=1}^{m} R\left(\alpha_{i}, \bar{\alpha}_{i}, \alpha_{j}, \bar{\alpha}_{j}\right) \leq b\left[\left(\sum_{i=1}^{m}\left\|\alpha_{i}\right\|^{2}\right)^{2}+\sum_{i=1}^{m}\left\|\alpha_{i}\right\|^{4}\right] .
$$


Proof of Theorem 1. Let $D$ be a smooth, ample divisor in $M$. Then, there exists an integer $\alpha$ such that

$$
c_{1}\left(K_{M}\right)=\alpha c_{1}([D]) .
$$

If $K_{M}$ is not ample, then $\alpha \leq 0$. It then follows from the third author's solution of the Calabi conjecture that there exists a Kähler metric $\omega^{\prime}$ on $M$ whose Ricci curvature is nonnegative. We shall prove that $\omega^{\prime}$ is not compatible with $\omega$.

Let $R_{i \bar{j}}$ and $R_{i \bar{j} k \bar{l}}$ denote, respectively, the Ricci curvature tensor and the curvature tensor of $\omega$. Similarly, we denote by $R_{i \bar{j}}^{\prime}$ and $R_{i \bar{j} k \bar{l}}^{\prime}$, respectively, the Ricci curvature tensor and the curvature tensor of $\omega^{\prime}$. Let

$$
S=\frac{n\left(\omega^{\prime}\right)^{n-1} \wedge \omega}{\left(\omega^{\prime}\right)^{n}}=\sum_{i, j=1}^{n} g^{\prime i \bar{j}} g_{i \bar{j}}
$$

Here we locally write

$$
\omega=\frac{\sqrt{-1}}{2} \sum_{i, j} g_{i \bar{j}} d z^{i} \wedge d \bar{z}^{j}, \quad \omega^{\prime}=\frac{\sqrt{-1}}{2} \sum_{i, j} g_{i \bar{j}}^{\prime} d z^{i} \wedge d \bar{z}^{j}
$$

where $\left(g^{i \bar{j}}\right)$ denotes the transposed inverse of $\left(g_{i \bar{j}}\right)$, and similarly for $\left(g^{\prime i \bar{j}}\right)$.

Let us compute $\Delta^{\prime} S$, where $\Delta^{\prime}$ denotes the Laplacian associated with $\omega^{\prime}$. For convenience, we choose a normal coordinate system $\left\{z^{1}, \ldots, z^{n}\right\}$ near a point $x \in M$ such that

$$
g_{i \bar{j}}(x)=\delta_{i j}, \quad \frac{\partial g_{i \bar{j}}}{\partial z^{k}}(x)=0,
$$

and that

$$
g_{i \bar{j}}^{\prime}(x)=\delta_{i j} g_{i \bar{i}}^{\prime}(x)
$$

Then, as in [7. p. 371], we assert that

$$
\Delta^{\prime} S=\sum_{i} \frac{R_{i \bar{i}}^{\prime}}{\left(g_{i \bar{i}}^{\prime}\right)^{2}}+\sum_{i, j, k} \frac{\left|\partial g_{i \bar{j}}^{\prime} / \partial z^{k}\right|^{2}}{g_{i \bar{i}}^{\prime}\left(g_{j \bar{j}}^{\prime}\right)^{2} g_{k \bar{k}}^{\prime}}-\sum_{i, k} \frac{R_{i \bar{i} k \bar{k}}}{g_{i \bar{i}}^{\prime} g_{k \bar{k}}^{\prime}} \quad \text { at } x .
$$

For completeness, this assertion will be proved at the end. The assertion implies that

$$
\Delta^{\prime} S \geq-\sum_{i, k} \frac{R_{i \bar{i} k \bar{k}}}{g_{i \bar{i}}^{\prime} g_{k \bar{k}}^{\prime}}
$$

Now we are in a position to apply Lemma 2.1 Let

$$
\alpha_{i}=\left(g_{i \bar{i}}^{\prime}\right)^{-1 / 2} \frac{\partial}{\partial z^{i}}, \quad i=1, \ldots, n .
$$


Then, $\alpha_{1}, \ldots, \alpha_{n}$ are orthogonal tangent vectors in $T_{x} M$. It follows that

$$
\begin{aligned}
\sum_{i, k} \frac{R_{i \bar{i} k \bar{k}}}{g_{i \bar{i}}^{\prime} g_{k \bar{k}}^{\prime}} & =\sum_{i, k} R\left(\alpha_{i}, \bar{\alpha}_{i}, \alpha_{k}, \bar{\alpha}_{k}\right) \\
& \leq-\frac{\kappa}{2}\left[\left(\sum_{i=1}^{m}\left\|\alpha_{i}\right\|^{2}\right)^{2}+\sum_{i=1}^{m}\left\|\alpha_{i}\right\|^{4}\right] \\
& \leq-\frac{\kappa}{2}\left(1+\frac{1}{n}\right)\left(\sum_{i=1}^{m}\left\|\alpha_{i}\right\|^{2}\right)^{2} \\
& =-\frac{\kappa(n+1)}{2 n} S^{2},
\end{aligned}
$$

where $\kappa=\kappa(x) \geq 0$ is a constant depending only on the upper bound of the holomorphic sectional curvature at $x$. Therefore, we obtain that

$$
\Delta^{\prime} S \geq \frac{\kappa(n+1)}{2 n} S^{2} \geq 0
$$

By the maximum principle, the function $S$ must be identically equal to a (positive) constant. In particular, $\Delta^{\prime} S \equiv 0$ on $M$. Now suppose that the holomorphic sectional curvature is strictly negative at a point $x_{0}$. That is,

$$
-\kappa\left(x_{0}\right) \equiv \sup _{\eta \in T_{x_{0}} M \backslash\{0\}} \frac{R(\eta, \bar{\eta}, \eta, \bar{\eta})}{\|\eta\|_{g}^{4}}<0 .
$$

Apply (2.3) to $x_{0}$ and then combine with Lemma 2.1 to obtain that

$$
0=\Delta^{\prime} S\left(x_{0}\right) \geq \frac{\kappa\left(x_{0}\right)(n+1)}{2 n} S^{2}\left(x_{0}\right) \geq 0 .
$$

This implies that $S \equiv S\left(x_{0}\right)=0$, which is a contradiction. This proves Theorem 1, except for verifying the assertion (2.3).

Let us now prove the assertion

$$
\Delta^{\prime} S=\sum_{i} \frac{R_{i \bar{i}}^{\prime}}{\left(g_{i \bar{i}}^{\prime}\right)^{2}}+\sum_{i, j, k} \frac{\left|\partial g_{i \bar{j}}^{\prime} / \partial z^{k}\right|^{2}}{g_{i \bar{i}}^{\prime}\left(g_{j \bar{j}}^{\prime}\right)^{2} g_{k \bar{k}}^{\prime}}-\sum_{i, k} \frac{R_{i \bar{i} k \bar{k}}}{g_{i \bar{i}}^{\prime} g_{k \bar{k}}^{\prime}}
$$

in which we use the normal coordinate chart satisfying (2.1) and (2.2). For simplicity, we denote

$$
\partial_{i}=\frac{\partial}{\partial z^{i}}, \quad \partial_{\bar{j}}=\frac{\partial}{\partial \bar{z}^{j}}, \quad \text { for all } 1 \leq i, j \leq n ;
$$

we shall use the summation convention unless otherwise indicated. Note that at the point $x$,

$$
\begin{aligned}
\Delta^{\prime} S & =g^{\prime k \bar{l}} \partial_{k} \partial_{\bar{l}}\left(g^{\prime i \bar{j}} g_{i \bar{j}}\right) \\
& =g^{\prime k \bar{l}} g_{i \bar{j}} \partial_{k} \partial_{\bar{l}} g^{\prime i \bar{j}}+g^{\prime k \bar{l}} g^{\prime i \bar{j}} \partial_{k} \partial_{\bar{l}} g_{i \bar{j}} .
\end{aligned}
$$

Observe that, by using (2.1), we have

$$
R_{i \bar{j} k \bar{l}}(x)=-\partial_{k} \partial_{\bar{l}} g_{i \bar{j}}(x) .
$$

Then, the second term on the right of (2.5) is equal to

$$
-\sum_{i, k} \frac{R_{i \bar{i} k \bar{k}}}{g_{i \bar{i}}^{\prime} g_{k \bar{k}}^{\prime}},
$$


in which (2.2) is used. It remains to show that

$$
g^{\prime k \bar{l}} g_{i \bar{j}} \partial_{k} \partial_{\bar{l}} g^{\prime i \bar{j}}=\sum_{i} \frac{R_{i \bar{i}}^{\prime}}{\left(g_{i \bar{i}}^{\prime}\right)^{2}}+\sum_{i, j, k} \frac{\left|\partial g_{i \bar{j}}^{\prime} / \partial z^{k}\right|^{2}}{g_{i \bar{i}}^{\prime}\left(g_{j \bar{j}}^{\prime}\right)^{2} g_{k \bar{k}}^{\prime}} .
$$

Notice that

$$
\begin{aligned}
g^{\prime k \bar{l}} g_{i \bar{j}} \partial_{k} \partial_{\bar{l}} g^{\prime i \bar{j}}= & -g^{\prime k \bar{l}} g_{i \bar{j}} \partial_{k}\left(g^{\prime i \bar{q}} g^{\prime p \bar{j}} \partial_{\bar{l}} g_{p \bar{q}}^{\prime}\right) \\
= & g^{\prime k \bar{l}} g_{i \bar{j}}\left(g^{\prime i \bar{b}} g^{\prime a \bar{q}} g^{\prime p \bar{j}} \partial_{k} g_{a \bar{b}}^{\prime}+g^{\prime i \bar{q}} g^{\prime p \bar{b}} g^{\prime a \bar{j}} \partial_{k} g_{a \bar{b}}^{\prime}\right) \partial_{\bar{l}} g_{p \bar{q}}^{\prime} \\
& -g^{\prime k \bar{k}} g_{i \bar{j}} g^{\prime i \bar{q}} g^{\prime p \bar{j}} \partial_{k} \partial_{\bar{l}} g_{p \bar{q}}^{\prime} .
\end{aligned}
$$

Let us first handle the last term in (2.7). Recall that

$$
R_{k \bar{l} \bar{q}}^{\prime}=-\partial_{k} \partial_{\bar{l}} g_{p \bar{q}}^{\prime}+g^{\prime a \bar{b}} \partial_{k} g_{p \bar{b}}^{\prime} \partial_{\bar{l}} g_{a \bar{q}}^{\prime} .
$$

It follows that

$$
-g^{\prime k \bar{l}} \partial_{k} \partial_{\bar{l}} g_{p \bar{q}}^{\prime}=R_{p \bar{q}}^{\prime}-g^{\prime k \bar{l}} g^{\prime a \bar{b}} \partial_{k} g_{p \bar{b}}^{\prime} \partial_{\bar{l}} g_{a \bar{q}}^{\prime} .
$$

Substituting this into the last term in (2.7) yields that

$$
\begin{aligned}
g^{\prime k \bar{l}} g_{i \bar{j}} \partial_{k} \partial_{\bar{l}} g^{\prime i \bar{j}} & \\
= & g_{i \bar{j}} g^{\prime k \bar{k}} g^{\prime i \bar{b}} g^{\prime a \bar{q}} g^{\prime p \bar{j}} \partial_{k} g_{a \bar{b}}^{\prime} \partial_{\bar{l}} g_{p \bar{q}}^{\prime}+g_{i \bar{j}} g^{\prime k \bar{l}} g^{\prime i \bar{q}} g^{\prime p \bar{b}} g^{\prime a \bar{j}} \partial_{k} g_{a \bar{b}}^{\prime} \partial_{\bar{l}} g_{p \bar{q}}^{\prime} \\
& +g_{i \bar{j}} g^{\prime i \bar{q}} g^{\prime p \bar{j}} R_{p \bar{q}}^{\prime}-g_{i \bar{j}} g^{\prime i \bar{q}} g^{\prime p \bar{j}} g^{\prime k \bar{l}} g^{\prime a \bar{b}} \partial_{k} g_{p \bar{b}}^{\prime} \partial_{\bar{l}} g_{a \bar{q}}^{\prime} \\
= & g_{i \bar{j}} g^{\prime k \bar{l}} g^{\prime i \bar{b}} g^{\prime a \bar{q}} g^{\prime \prime \bar{j}} \partial_{k} g_{a \bar{b}}^{\prime} \partial_{\bar{l}} g_{p \bar{q}}^{\prime}+g_{i \bar{j}} g^{\prime i \bar{q}} g^{\prime p \bar{j}} R_{p \bar{q}}^{\prime} \\
= & \sum_{i, k, a} \frac{\left|\partial_{k} g_{a \bar{i}}^{\prime}\right|^{2}}{\left(g_{i \bar{i}}^{\prime}\right)^{2} g_{k \bar{k}}^{\prime} g_{a \bar{a}}^{\prime}}+\sum_{i} \frac{R_{i \bar{i}}^{\prime}}{\left(g_{i \bar{i}}^{\prime}\right)^{2}} .
\end{aligned}
$$

This verifies (2.6). Hence, the assertion is proved. This finishes the proof of Theorem 1.

\section{REFERENCES}

[1] C. Birkenhake and H. Lange, Complex Tori, Birkhäuser, 1999. MR1713785 (2001k:14085)

[2] F. Campana, Twistor spaces and nonhyperbolicity of certain symplectic Kähler manifolds, Complex analysis (Wuppertal, 1991), 64-69, Aspects Math., E17, Vieweg, Braunschweig, 1991. MR1122161 (92j:32092)

[3] S. Kobayashi, Hyperbolic Complex Spaces, Springer, 1998. MR.1635983 (99m:32026)

[4] T. Peternell, Calabi-Yau manifolds and a conjecture of Kobayashi, Math. Z. 207 (1991), 305-318. MR:1109668 (92g:14039)

[5] H. Royden, The Ahlfors-Schwarz lemma in several complex variables, Comment. Math. Helvetici 55 (1980), 547-558. MR604712 (82i:32049)

[6] P. M. H. Wilson, Calabi-Yau manifolds with large Picard number, Invent. Math. 98 (1989), 139-155. MR1010159(90h:14044)

[7] $\mathrm{D}$. Wu, S. T. Yau and F. Zheng, A degenerate Monge-Ampère equation and the boundary classes of Kähler cones, Math. Res. Lett. 16 (2009), 365-374. MR2496750 (2010c:32061)

[8] S. T. Yau, A general Schwarz lemma for Kähler manifolds, Amer. J. Math. 100 (1978), 197-203. MR0486659 (58:6370) 
Department of Mathematics, University of Notre Dame, Notre Dame, Indiana 46556

E-mail address: pmwong@nd.edu

Department of Mathematics, The Ohio State University, 1179 University Drive, NEWARK, OHIO 43055

E-mail address: dwu@math.ohio-state.edu

Department of Mathematics, Harvard University, One Oxford Street, Cambridge, MASSACHUSETTS 02138

E-mail address: yau@math.harvard.edu 